\title{
Study of the Human Eye Working Principle: An impressive high angular resolution system with simple array detectors
}

\author{
Diego Betancourt and Carlos del Río \\ Antenna Group, Public University of Navarra, Campus Arrosadia s/n 31006 Pamplona, Spain \\ carlos@unavarra.es
}

\begin{abstract}
In this paper the working principle of the human eye is studied since uses very simple detectors, cones and rods, to provide an impressive high angular resolution without using any kind of scanning technique. Usually the resolution capability of the human eyes has been attributed to the complexity of the brain, but it is quite difficult to understand that something, specially small details, that it has not been seen by the eyes could be generated by the brain.

Finally, it will be proved that with the actual technology and design philosophy of array antenna systems, the maximum achievable angular resolution of a realistic antenna array system is seriously limited and it is equivalent to the one that it could have an insect with compound eyes. It is clear the human eye is able to overcome this limit, and the idea is to learn how to introduce this to the design procedure of antenna array systems.
\end{abstract}

\section{Introduction}

One of the most important requirements of all the antenna systems is the angular resolution achievable, to be able to distinguish two punctual sources placed quite close to each other in far field. The angular resolution is determined by the possibility of having high directive beams quite close, in angle, to each other.

With the actual technologies, the antenna systems having one or more high directive beams at microwave and millimeter wave ranges could be classified in two kinds of systems: a).- systems with a unique lens or focusing device, and b).- systems with multiple lenses.

In the first case, the possibility to increase the number of beams passes by admitting some kind of distortion of the beams placed out of focus. Practically all the more commonly used antenna systems are included here, some of them with more focusing capabilities in the lens or focusing device and some others with more directive radiating elements. The classical parabolic reflector antenna with the low directive feeder could be an excellent example of this type of systems, but also the multi-feed reflector antenna configuration.

The HFI and LFI experiments of PLANCK [1] use this configuration of multiple feeds and multiple frequencies illuminating only a unique reflector system (Fig.1), and equally, every one of the radiating elements of ALMA [2] and VLA [3] experiments.
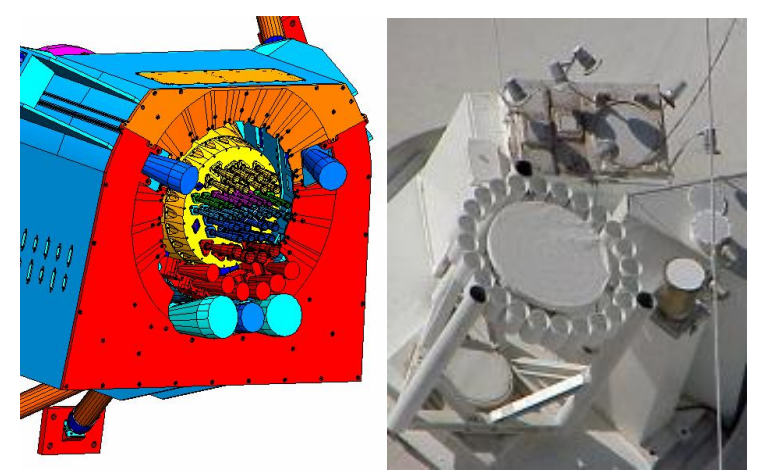

Figure 1.- Multiple feed disposition at the focal plane of the PLANCK (left) and VLA (right) systems.

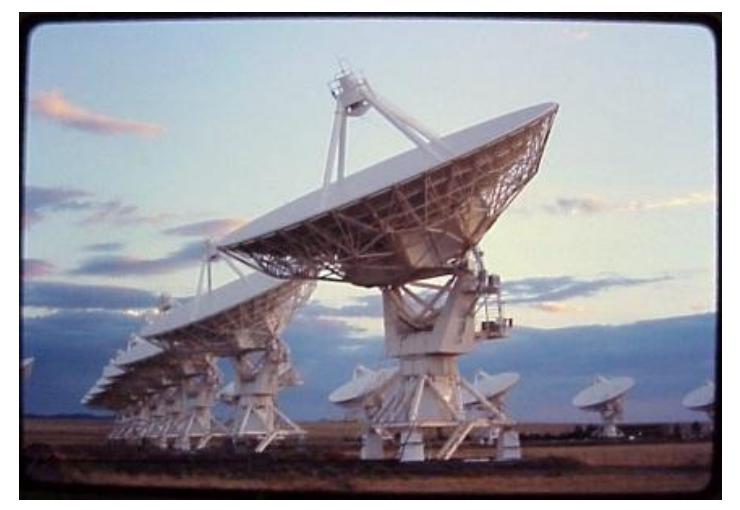

Figure 2.- VLA system picture. Each reflector includes at its focal plane the multiple feed configuration shown in figure 1. 
The second case is the case of the big arrays configurations (Fig.2), where the radiating elements are complete antenna systems disposed over a large area with a special lattice. Examples as ALMA [2], VLA [3], etc, are well known by all the scientific community.

However, as it is very well known, in both cases, the main problem is the angular resolution achievable, since there is no possible to reduce the distance between high directive beams below the aperture dimensions of the radiating elements.

The limitation of having a small number of detectors per lens reduces the possibilities of natural extension of the actual technology to handle a large number of detectors (Fig. 3). In front of that limit, the only valid solution, to enlarge the system, is to iterate the full system itself, obtaining some kind of compound eye of some insects, where there are a lot of small antennas (detectors) focused by its own lens (ommatidia), conform a complete visual system (Fig. 4).

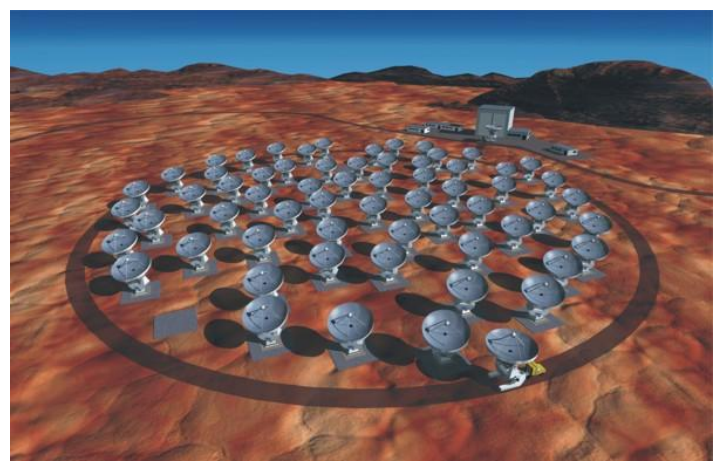

Figure 3. Artist view of the ALMA experiment

Of course, due to the size of the different receiving elements (small lenses in the case of the compound eyes of the insects) the angular resolution is equally limited.

Nevertheless, in the nature there are some examples of antenna systems with impressively high resolution. One of the most extended is the human eye that it is working at optical frequency range and the achievable resolution is really impressive.

This paper want to be an invitation to study the working principle of these kind of eyes as detectors, to consider the viability of applications of these principles to the antenna system design at lower frequencies.

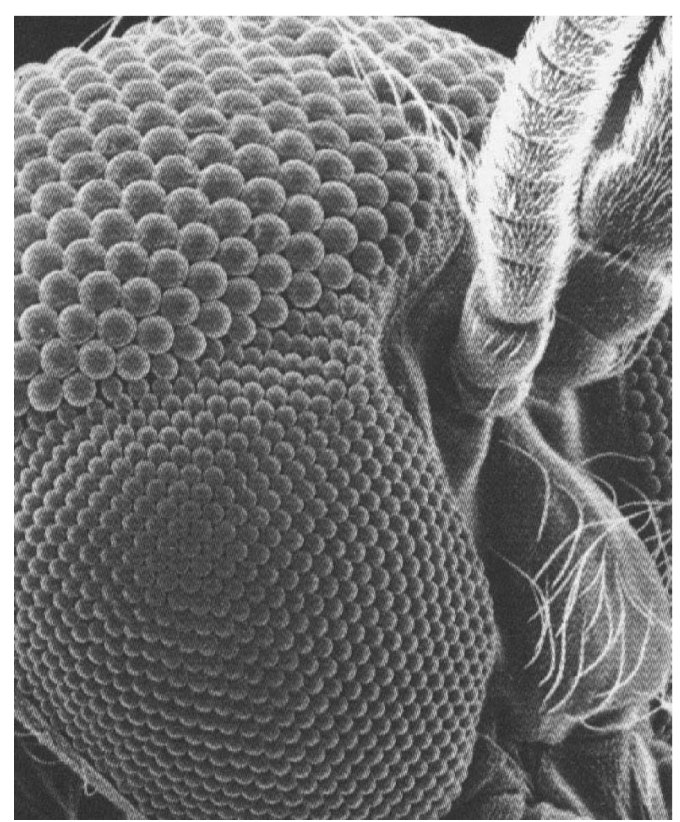

Figure 4. Close view of the compound eye of an insect.

\section{The human eye}

The human eyes have an impressive performance. They are able to focus near and far objects automatically; they have possibilities to see under bad circumstances (low light); they are able to control de entrance of light to prevent possible saturation of photoreceptors; and finally, the most impressive feature, the really high resolution of the obtained images.

But, it is even more impressive if we try to understand the human eye under antenna parameters. To be able to distinguish two points quite close to each other at certain distance, we should need some kind of really high directivity. In some experiments performed shown that the needed directivities should be close to $90 \mathrm{~dB}$, which means, that the main beam is subtended under angles of less that 1 minute of arc. But this is even more amazing if we think that many other beams should be simultaneously placed every 1 minute of arc, to be able to distinguish the changes of light intensity and color.

Some people like to assign to the brain the capabilities to obtain such high resolution images, but it is clear that it could be difficult to "generate" small details that have never been received by the eye. It could be understandable some kind of interpolating post-processing technique to try to solve points placed between photoreceptors to obtain a continuity sensation 
of the images, but this never would generate additional details in the image increasing the resolution.

Thus, it is clear that some more investigation about the human eye could be very interesting to try to clarify these aspects that seems be not so clear.

First of all, we will collect some data from the human eye in order to illustrate the working principle of the human vision, and to evaluate the causes of the high resolution.

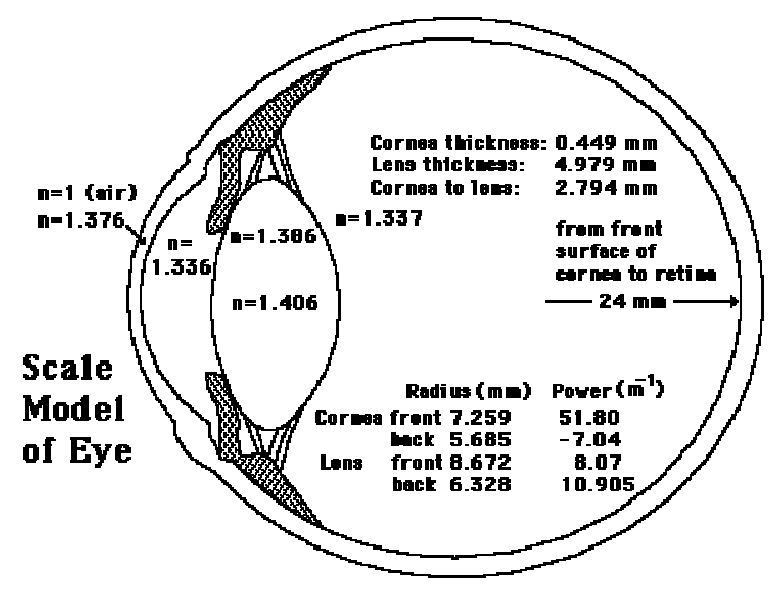

Figure 5.- Scale model of the human eye, including the different lenses and their refractive indices.

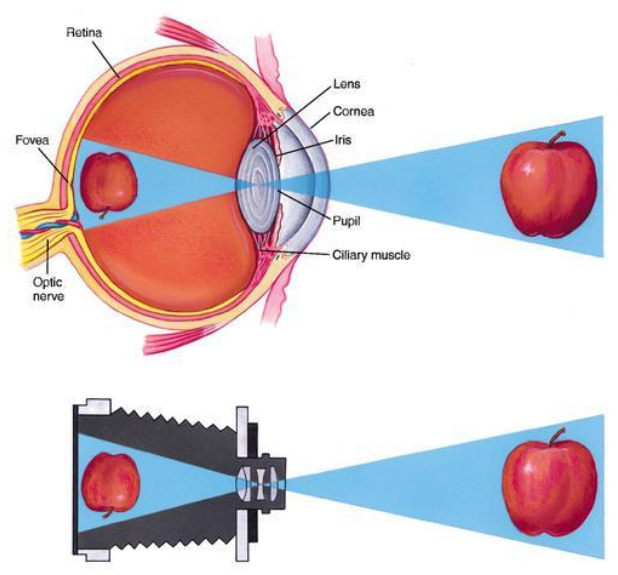

Figure 6.- Optical focusing system of the human eye and a photographic camera, showing the similarities in the inverted projected image over the retina or the film respectively.

In principle, in the human eye, the light passes through the cornea and the crystalline having a focus situated just over the retina. The total refractive power of the human eye is measured in dioptres and it is determined by the inverse of the focal length, being 62 the considered normal value for a current human eye [4]. This means that the focal length is then $16 \mathrm{~mm}$, and it corresponds with the separation between the crystalline and the retina (Fig. 5). Then, the images in front of the eyes (just at the focal plane outside the eye) will be inversely projected over the retina as in a photographic camera (Fig. 6).

Over the retina there are special cells, photoreceptors, specially prepared to receive the information modulated at optical frequencies introducing this information in the neural system to the brain to process. There are different kinds of photoreceptors: cones and rods. The cones responsible of the colored vision and the rods more related with the vision under poor lighting conditions.

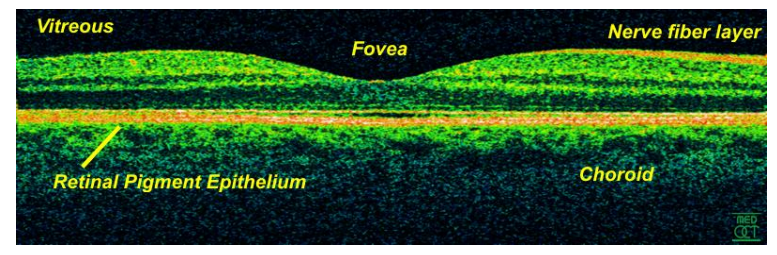

Figure 7.- Optical Coherence Tomography at $800 \mathrm{~nm}$ of the Macula in a human retina.

It is also well known that we really have properly focused a small cone subtended in an angle of less than one degree, and the responsible of this is a small area on the retina just centered at the vision axis called Macula recognized as a depression of the retina surface being the deeper point knows as Fovea (Fig. 7).

In the Macula, the density of cones is 160.000 per square millimeter being the main responsible of the focused central vision. The Macula is in fact acting like a divergence lens, dispersing the parallel rays arriving close to the optical axis over a wider area of receptors. This divergence effect is not included properly in the optical system of the eye since the Fovea is only $100 \mu \mathrm{m}$ deep, and the dispersion effect is really small and only relevant for the increase of area of receptors affected.

It is also known that the cones diameter is approximately $1.5 \mu \mathrm{m}$ and the separation between two cones is about $0.5 \mu \mathrm{m}$.

With these dimensions, if we apply the photography principle and we expect to obtain focused over the retina the inverted image, we could calculate the resolution of the retina image simply translating the separation between two cones outside the eye. To solve 
two different points outside, the retinal image should have an "unexcited" cone between two other excited ones by the two points respectively, given a distance of $4 \mu \mathrm{m}$ as the minimum distance necessary to solve two points on the retina. Translating this distance out of the eye up to a distance of $350 \mathrm{~mm}$ (reading distance), these resolution step is $84 \mu \mathrm{m}$.

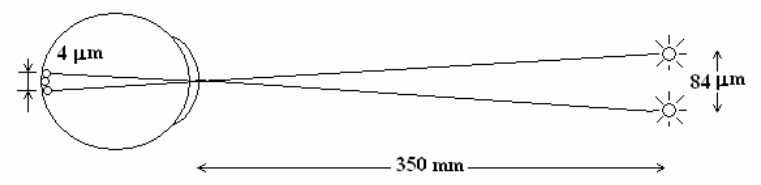

Figure 8.- First approximation of human eye resolution.

This could mean that we shouldn't be able to distinguish anything smaller than this resolution step, but really we are able to see below that limit, so something more should be consider.

\section{Explanation for the higher angular resolution of the human eye}

Some authors suggest that there is a very quick and small oscillation movement of the eyes that could help to refill the empty spaces between cones.

At that point of the explanation it is important to think a little bit about the detection mechanism. The cones are not detecting the carrier signal (optical frequencies); they are really detecting the modulated signal. Certainly, each type of cone has its own frequency response to optical frequencies, being more sensible to different colors: red, green and blue.

In a simplified model, we could think that the cones could be acting as integrators, just counting the number of photons received, and generating an output signal level proportional to that number.

Under the assumption of the rapid and small vibration of the eye to increase the resolution, it could be argued that it could really difficult to think that the brain could know exactly the position of the eye when each photon arrives to each cone to reconstruct the higher resolution image. Assuming this integration behavior, this vibrating phenomenon could really diffuse the image over the retina.

Furthermore, under the optical theory, the light travels in straight lines, but really some diffraction should be consider since the light is also a wave. This means that really the light will not focus in a single point but in an area. This phenomenon again generates some diffusion of the retina image.

But even more, if we study the chemistry reactions of the reception mechanism of a photon by a cone, some horizontal coupling between neighboring cones have been reported [4], in some kind of amplifying effect ensuring that all photons are properly received by the retina, improving the signal-noise ratio of the receiving system.

All these three phenomena generate some kind of distortion in the retina image since the information of one photon is effectively spread over some area of the retina, either by the diffraction of light, by the movement of the eye or by the chemistry mechanisms.

So, in summary, where we were expecting to obtain an image perfectly focused over the retina, since is placed just at the focal plane of the eye lenses (cornea and crystalline), we are really obtaining a totally defocused image.

Originally, we start this study to explain the higher resolution of the human eye. Could all this really explain the higher resolution? Or, on the contrary we should continue investigating other causes to justify the higher resolution.

Let's follow a little bit more inside the composition of the retina. Looking carefully at the total composition of the retina, we found two additional layers of neurons over the light receptors highly interconnected (Fig. 9). These two layers could perform some kind of image processing trying to build a higher resolution image to be sent to the brain through the optical nerve.

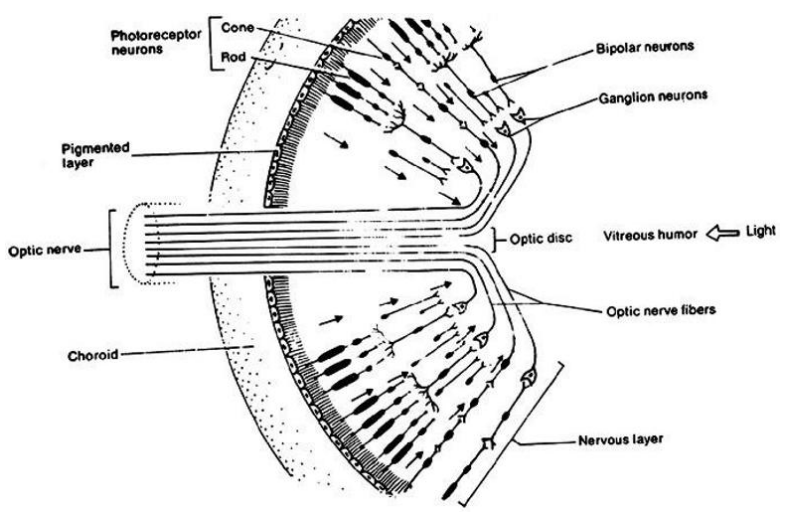

Figure 9.- Detailed disposition of the neurons and the photoreceptors inside the retina of a human eye. 
This could be possible thanks to the inherent coherence of the eye as a detector of photons. The effect of a photon over the retina finally excites a set of photoreceptor cells, as it was justified above. With the neural networks we could identify without any problem the impact point calculating the position of the maximum.

To do this in only one layer, we should have as much neurons as detecting cells over the retina, being every neuron connected to several retina cells (this number should corresponds with the number of excited cells by a single photon) and applying a threshold function.

Under the inherent coherence conditions of the detection mechanism, the linearity perfectly applies with any distortion phenomena, so by using a single layer of neurons we really could clarify the diffused retinal image, or even more, we could be able to increase the resolution of the received image having more neurons than cones.

There are some advantages of having a diffuse image over the retina to be clarified afterwards, but the most important is the robustness of the vision system is substantially improved since the information over the retina is redundant. By this method, it could be understandable that different types of cones and rods could be working together to define the small details of the image in front the eyes.

Coming back to the antenna design theory, it is clear that the retina could be consider as an antenna array, each of the cones acts as a detector, where to obtain a high directive beam, some area over the retina should be effectively used, having highly overlapped radiating areas that could justify very close high directive beams, and therefore, high angular resolution.

This is, every cone is used to define many different spots of the obtained image, or in other words we could also say that the information corresponding with every spot of the image have been effectively received by many cones, allowing a direct detection strategy with a reasonable signal to noise ratio, obviously obtaining a very simple detecting system.

\section{Conclusions}

In this paper, the behavior of the human eye as an antenna system have been analyzed noting the different solutions for the main common trade-offs of antenna array systems, in particular regarding the angular resolution and the signal to noise ratio.

Normally, to improve the angular resolution, more directive beams close to each other are needed. With the conventional techniques, the minimum distance between two neighboring beams is determined by the size of the radiating elements used to create them.

On the other hand, to solve to problem of signal to noise ratio, historically the heterodyne detection is preferred instead a direct detection, since the power balance of the system ensures more signal to noise ratio at the receptor position.

As we have seen, the human eye uses very different techniques to solve these problems. In fact, both problems are solved with the same strategy, using many photoreceptors, cones, to create each of the beams, and re-using many of these cones for other neighboring beams. Thanks to this recycling technique of the cones, the effective radiating areas are practically overlapped and since there are many cones detecting the same information coherently, the signal/noise ratio also improves.

The final result is well know for everyone and is that the "antenna system" of the human eye works really very well using very simple detection techniques. So the final question is: Could we use these techniques in microwave and millimeter imagining systems?; and, what about using these techniques in Smart antennas, MIMO, or any kind of array antenna configuration? With a high probability the answer could be that we could have very much simpler systems with very similar or improved performances just copying the detecting strategies of the human eye.

\section{Acknowledgements}

This work have supported by the Spanish Government by the project TIC2003-09317-C03-01.

\section{References}

[1] http://www.esa.int/science/planck

[2] http://www.alma.nrao.edu/

[3] http://www.nrao.edu/

[4] Kaufman, P.L. and Alm, A., "Adler's Physiology of the eye", Edited by P. Kaufman, Mosby, tenth edition, ISBN 0-323-01136-5. 